\title{
Relationship Between Clinical Disease Characteristics and Acute and Structural Changes in Sacroiliac Magnetic Resonance Imaging in Patients with Newly Diagnosed Axial Spondyloarthritis
}

\author{
(1) Mustafa Corum, (1) Tugba Aydin \\ Istanbul Physical Medicine and Rehabilitation Training and Research Hospital, Clinic of Physical Medicine and Rehabilitation, Istanbul, \\ Turkey
}

\section{Abstract}

\begin{abstract}
Aim: The association between acute and structural lesions on magnetic resonance imaging (MRI) of the sacroiliac joint (SIJ) with clinical and laboratory features has not been fully investigated. This study aimed to compare the clinical and laboratory features of patients diagnosed with axial spondyloarthritis (axSpA) with and without inflammation or structural lesions in SIJ MRI.
\end{abstract}

Methods: Eighty four patients diagnosed with axSpA between June 2019 and June 2020 were retrospectively examined. Patients' medical records, laboratory data and MRI findings were collected. Inflammation was measured by C-reactive protein levels and erythrocyte sedimentation rate (ESR) (Westergren method, 0-20 mm/h). Moreover, the Spondyloarthritis Research Consortium of Canada MRI index was used to assess inflammation and structural changes of the SIJ.

Results: ESR values were statistically significantly different in patients with SIJ backfill and ankylosis ( $p=0.013$ and $p=0.016$, respectively) and symptom duration in patients with SIJ erosion and ankylosis ( $p=0.048$ and $p=0.049$, respectively) compared to those without. Morning stiffness was the only IBP characteristic which had a significant correlation with the presence of SIJ ankylosis ( $p=0.048, r=-$ 0.218).

Conclusion: Clinical and laboratory characteristics in axSpA patients are associated with chronic structural lesions rather than inflammation signs in SIJ MRI.

Keywords: Back pain, inflammation, sacroiliac joint, spondyloarthritis

\section{Introduction}

Axial spondyloarthritis (axSpA) is a group of chronic inflammatory rheumatic diseases involving ankylosing spondylitis (AS), reactive arthritis, psoriatic arthritis, arthritis associated with inflammatory bowel disease and undifferentiated SpA (1). Genetics, environmental factors and immune-mediated mechanisms contribute to axSpA pathogenesis; however, its etiology is indefinite (2-4).

The modified New York criteria include only the presence of structural damage to sacroiliac joints (SIJ) in plain radiography; therefore, it can diagnose only advanced diseases (2). Because of the slow development of radiographic changes, only $70 \%$ of axSpA patients can meet the modified New York criteria after 5 years of symptoms (5). To facilitate early diagnosis and treatment of axSpA, the following criteria have been added to the classification of the Assessment of Spondyloarthritis International Society (ASAS): the presence of sacroiliitis on magnetic resonance imaging $(\mathrm{MRI})$, radiographs along with a SpA characteristic or positivity of human leukocyte antigen B27 (HLA B27) along with two SpA characteristics in patients with chronic back pain aged $<45$ years (6). AxSpA treatment is aimed at early intervention during the disease course, when the response to treatment is at its 
peak in the period before the occurrence of radiographic sacroiliitis (7).

Therefore, MRI is an important tool for SpA diagnosis because it can identify inflammation before the detection of structural radiographic changes (8). Furthermore, MRI scans can provide additional important objective information about treatment effectiveness (9). Bone marrow oedema (BME) of SIJ is a well-known inflammatory lesion in SIJ and can progress to chronic lesions such as $\mathrm{BME}$, subchondral sclerosis, erosions, fat metaplasia and ankylosis $(10,11)$.

Inflammatory back pain (IBP) is a chronic lower back pain which usually begins during young adulthood, improving by exercise and worsening with immobility and associated with morning stiffness. The presence of IBP characteristics, an important component of the ASAS classification criteria, has been specifically indicated for the diagnosis of axSpA in patients with chronic back pain (12). While the prevalence of IBP ranges between $5.0 \%$ and $6.0 \%$ among adults aged 20-69 years, the prevalence of SpA is estimated to be between $0.4 \%$ and $1.3 \%(13,14)$. This suggests that IBP characteristics can be common components of lower back pain due to many different diseases.

Therefore, this study aimed to determine which IBP parameters and disease characteristics are associated with acute (active inflammation) and/or chronic structural MRI changes in SIJ during the initial diagnosis of axSpA.

\section{Methods}

\section{Study Design}

The ethical approval was obtained from Bakirkoy Sadi Konuk Training and Research Hospital Ethics Committee (no: 2020/308). The study was conducted in accordance with the Helsinki Declaration and allowed the retrospective data collection, analysis of laboratory and MRI examinations and conducting phone calls.

The medical records of 110 patients with IBP, who were admitted to our department, were retrospectively examined and 84 patients newly diagnosed axSpA according to the ASAS criteria (1) upon their admittance to the outpatient clinic of Physical Medicine and Rehabilitation between June 2019 and June 2020 were included in the study. The medical records of the patients were obtained from the hospital database.

The inclusion criteria included meeting ASAS classification criteria [imaging criteria, sacroiliitis on imaging and $\geq 1 \mathrm{SpA}$ feature and clinical criteria, positive HLA-B27 and $\geq 2$ other SpA features] for axSpA as a result of retrospective examination of the patients' medical records, being diagnosed for the first time and obtaining the first MRI findings and laboratory data in our hospital. Also, patients were included aged between 18 and 65 years, had symptoms for $>3$ months and had initial diagnosis in our hospital. Exclusion criteria were the inability to contact with patients and incomplete data regarding symptoms.

\section{Clinical and Laboratory Assessment}

IBP parameters include pain duration of $>3$ months, pain during the second half of the night, morning stiffness, reduced pain with exercise and the onset of symptoms before 40 years of age. The IBP parameters were scored with "yes=1", "no=0", and the total IBP score (0-5) was calculated. Moreover, demographic data such as gender, age and symptom duration were recorded.

C-reactive protein (CRP) (immunoturbidimetric method, 0-0.5 mg/L) and erythrocyte sedimentation rate (ESR) (Westergren method, 0-20 mm/h) levels were measured. CRP level of $>0.5 \mathrm{mg} / \mathrm{L}$ and ESR level of $>20 \mathrm{~mm} / \mathrm{h}$ were defined as elevated CRP and ESR levels, respectively.

\section{MRI Assessment}

MRI examinations were performed on a 1.5 Tesla unit (MAGNETOM Aera; Siemens Healthcare, Erlangen, Germany) with a whole-body surface coil system in the supine position. All sequences were obtained using 12-15 semi-coronal slices with a thickness of $4 \mathrm{~mm}$ in the coronal oblique plane parallel to the long axis of the sacrum. The imaging protocol included T1-weighted spin-echo (T1W/ $\mathrm{SE})$ pulse sequence, T2-weighted turbo spin-echo (T2W/ TSE) sequence and short-tau inversion recovery (STIR) sequences. All images were evaluated by an experienced radiologist based on the ASAS classification criteria and the Spondyloarthritis Research Consortium of Canada (SPARCC) classification. The SPARCC scoring was used in the inflammatory and structural evaluation of SIJ (15).

An inflammatory lesion is defined as an increased bone marrow signal of either the iliac or sacral bones on the STIR sequence (Figure 1). The T1WSE MRI sequence may reveal a lesion in bone marrow adjacent to the subchondral bone, termed fat metaplasia characterized by a homogeneous increase in marrow signal, indicative of lipid accumulation and a distinct border (Figure 2A). Erosion is considered present when there is a breach in the cortical bone of either the iliac or sacral bones, which appears dark on both T1W/SE and STIR sequences, together with loss of the bright signal from the adjacent marrow matrix on T1W/SE MRI (Figure 2A). New tissue growth in erosive regions with the same signal intensity as fat metaplasia in T1W/SE MRI is called backfill (Figure 2B). Also, ankylosis is defined as a bone marrow signal on the T1W/SE sequence extending between the sacral and iliac bone marrow with a full-thickness loss of the dark 
appearance of the iliac and sacral cortical bone (Figure 2B).

The SPARCC inflammation score was based on the assessment of all signal changes in the sacrum until the iliac bone and sacrum foramina in the STIR sequence in six consecutive semi-coronal slices covering the cartilaginous part of the joint from posterior to anterior regions. Each SIJ is divided into 4 quadrants (upper and lower iliac and upper and lower sacral), each of which was evaluated separately. For the calculation of the SPARCC score of active inflammatory changes, the presence of hyperintensity lesions in the STIR sequence in each of these 4 quadrants was recorded in each of the 6 segments $(0=$ normal signal, $1=$ present lesion of increased intensity). Moreover, an additional point was given to the presence of an intense signal in the lesion in the 6 segments of each quadrant or the presence of a lesion with a depth of $\geq 1 \mathrm{~cm}$ (maximum total score=72) (16). SPARCC MRG SIJ structural score allows T1W/SE sequence MRI assessment of the presence

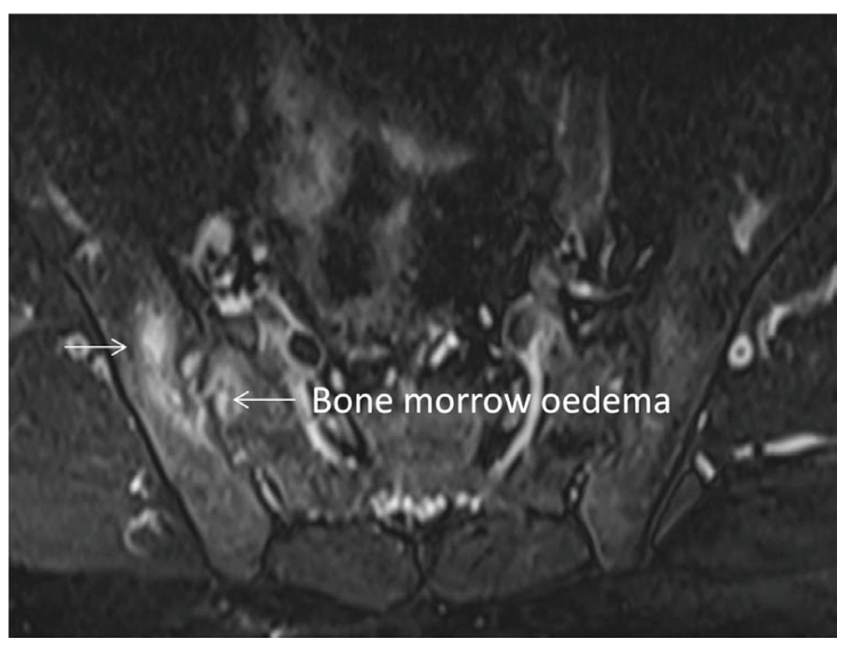

Figure 1. MRI findings of inflammatory lesions in the STIR sequence of SIJ

MRI: Magnetic resonance imaging, SIJ: Sacroiliac joint, STIR: Sequence and short-tau inversion recovery

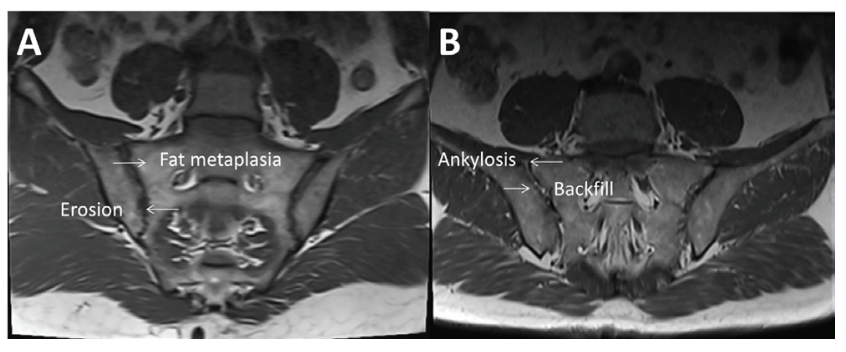

Figure 2. MRI findings of structural lesions in the T1W/SE sequence of SIJ. A) fat metaplasia and erosion B) backfill and ankylosis

MRI: Magnetic resonance imaging, SIJ: Sacroiliac joint, T1W/SE: T1weighted spin-echo of fat metaplasia, erosion, backfill and ankylosis. Five consecutive semi-coronal slices were evaluated from the posterior to the anterior regions, beginning from the transitional slice which is the first ligamentous cartilage slice. Each erosion and fat metaplasia was scored in total between 0-40 for 5 segments in 4 quadrants in SIJ, 0-8 per segment, whereas each backfill and ankylosis was scored a total of 0-20 for 5 segments, 0-4 per segment in 2 halves (upper versus lower) (11).

\section{Statistical Analysis}

The Statistical Package for the Social Sciences (Version 22.0, IBM Corp., Armonk, NY, USA) was used to analyze the data. The descriptive statistics of the data were expressed in mean values and standard deviation for continuous variables, and in counts and percentages for categorical variables. The Shapiro-Wilk test was used to determine whether the normal distribution was present. Spearman's correlation test was performed to analyze the relationship between IBP parameters, inflammation and structural lesions in SIJ. Differences in demographics and baseline disease characteristics were analyzed by the presence or absence of SIJ BME $(\geq 2$ or $0<2)$ and by the separate presence or absence of any structural lesions ( $>$ or $=0$ ). These analyses were performed using the MannWhitney $U$ test. Statistical significance was considered at $p<0.05$.

\section{Results}

Demographic, clinical, laboratory and SIJ MRI findings of patients are summarized in Table 1. A total of 84 patients were diagnosed with axSpA (35 females, 49 males; mean age $29.4 \pm 5.0$ years) and were included in the final analysis after exclusion of 26 patients due to inadequate clinical data and unwillingness to participate. The mean duration of symptoms for these patients was $3.3 \pm 2.3$ years. CRP elevation was detected in 18/84 patients (21.4\%) and erythrocyte sedimentation rate (ESR) elevation in $7 / 84$ patients (8.3\%), whereas the mean CRP and ESR values were $0.5 \pm 1.0 \mathrm{mg} / \mathrm{L}$ and $8.0 \pm 7.8 \mathrm{~mm} / \mathrm{h}$, respectively. A total of $73 / 84$ patients (86.9\%) had acute inflammation in the MRI, whereas certain patients had structural fat metaplasia (72/84, 85.7\%), erosion (78/84, 92.8\%), backfill (74/84, 88.0\%) and ankylosis (69/84, 82.1\%).

Table 2 shows the demographic and baseline disease characteristics in patients with BME (score $\geq 2$ ) and without BME (score <2) in SIJ MRI and the presence (score $>0$ ) or absence (score $=0$ ) of structural lesions. Similar differences were observed in demographic and basic disease characteristics when SIJ was characterized by the presence or absence of BME $(p>0.05)$. When it 
was characterized by patients with and without structural lesions in SIJ, the symptom duration was slightly statistically longer in SIJ erosion and ankylosis ( $p=0.048$ and $p=0.049$, respectively). The ESR values were statistically significant differences in SIJ backfill and ankylosis ( $p=0.013$ and $p=0.016$, respectively).

The association of different IBP characteristics with MRI findings (BME and structural lesions) is detailed in Table 3. Morning stiffness showed a slightly significant correlation with the presence of SIJ ankylosis $(p=0.048$, $r=-0.218$ ), there was no significant correlation between other IBP characteristics and acute and structural lesions in SIJ MRI.

\section{Discussion}

\begin{tabular}{|c|c|}
\hline Characteristics & Mean \pm SD or $n(\%)$ \\
\hline Age, years & $29.4 \pm 5.0$ \\
\hline Gender, female/male & $35(41.7) / 49(58.3)$ \\
\hline Duration of symptoms, years & $3.3 \pm 2.3$ \\
\hline $\mathrm{CRP}, \mathrm{mg} / \mathrm{L}$ & $0.5 \pm 1.0$ \\
\hline CRP elevation, yes/no & $18(21.4) / 66(78.6)$ \\
\hline $\mathrm{ESR}, \mathrm{mm} / \mathrm{h}$ & $8.0 \pm 7.8$ \\
\hline ESR elevation, yes/no & $7(8.3) / 77(91.7)$ \\
\hline Inflammatory back pain score (0-5) & $4.0 \pm 0.7$ \\
\hline SPARCC score of BME (0-72) & $18.4 \pm 14.7$ \\
\hline SPARCC score of fat metaplasia (0-40) & $13.0 \pm 9.3$ \\
\hline SPARCC score of erosion (0-40) & $13.1 \pm 9.1$ \\
\hline SPARCC score of backfill $(0-20)$ & $7.2 \pm 5.4$ \\
\hline SPARCC score of ankylosis (0-20) & $8.0 \pm 5.7$ \\
\hline \multicolumn{2}{|c|}{$\begin{array}{l}\text { Means (SD) is given for continuous variables; } n(\%) \text { is given for categorical data. } \\
\text { SD: Standard deviation, SPARCC: SPondyloArthritis Research Consortium of } \\
\text { Canada, BME: Bone marrow edema, CRP: C-reactive protein, ESR: Erythrocyte } \\
\text { sedimentation rate, SIJ: Sacroiliac joint }\end{array}$} \\
\hline
\end{tabular}

This study compares age, pain duration, inflammatory markers (CRP and ESR), IBP characteristics and patients with and without acute and structural lesions of SIJ MRI during the initial diagnosis of axSpA; thus, the study evaluates the relationship between the disease characteristics and SIJ MRI signs. According to the results of our study, the mean ESR values were higher than those in patients with SIJ backfill and ankylosis lesions and mean symptom duration was longer in patients with SIJ erosion and ankylosis lesions than those who did not. Furthermore, morning stiffness was the only IBP characteristic that had a significant correlation with the presence of ankylosis.

Although IBP characteristics are included in different diagnostic criteria used in both research and clinical practice for the diagnosis of axSpA, the strength of the relationship between IBP characteristics and the presence of axSpA disease is controversial (11). Although the underlying pathogenesis of IBP is not fully understood, it is believed that the inflammation in the MRI in SIJ and surrounding structures clinically represents IBP (17). However, studies have demonstrated that the acute lesion rate in SIJ MRI in patients with IBP characteristics is between $26 \%$ and $41 \%(18,19)$. Therefore, Braun et al. (20) emphasized that only IBP characteristics of limited value for the axSpA diagnosis and that imaging with IBP parameters or combination with other diagnostic criteria parameters can be useful. However, the relationship between acute and structural lesions in SIJ MRI and IBP has been investigated in a limited number of studies. Kivity et al. (17) examined the relationship between acute and structural sacroiliitis in MRI with IBP features and revealed ta significant correlation between BME with night pain and morning stiffness, as well as the relationship between nocturnal pain with structural lesions. Because of the connection between IBP features and the acute inflammation in SIJ MRI in the aforementioned study, it has been emphasized

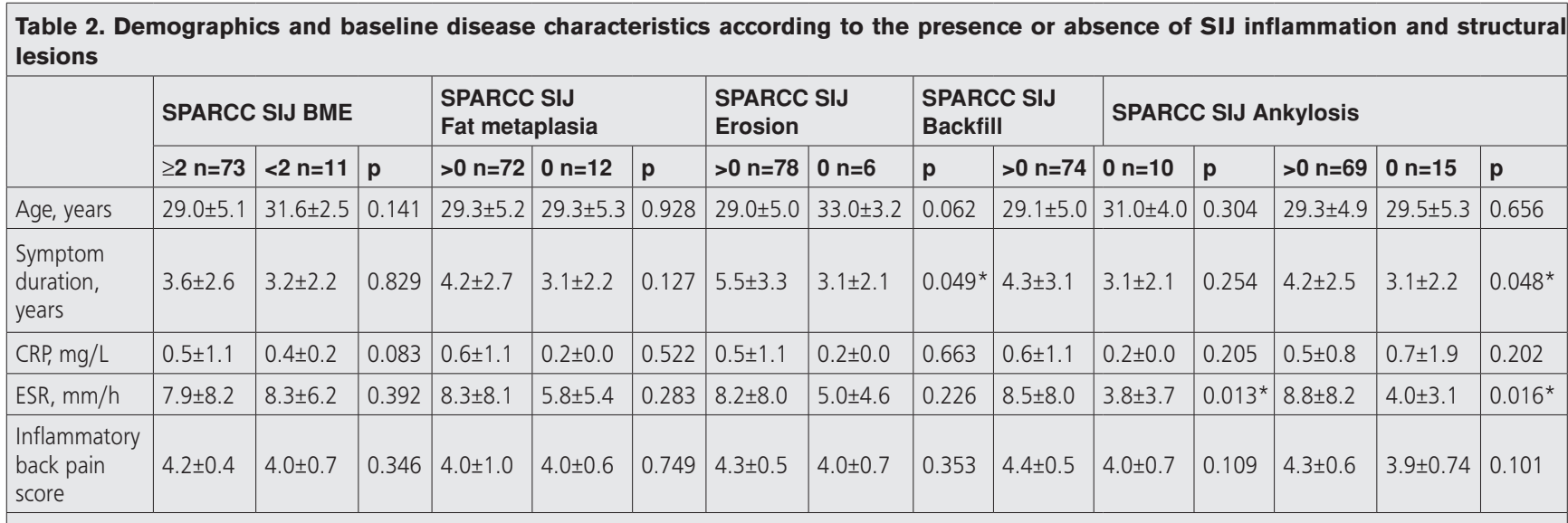

Values are mean \pm standard deviation. PS were calculated using Mann-Whitney U test. SPARCC: SPondyloArthritis Research Consortium of Canada, SIJ: Sacroiliac joint, BME: Bone marrow edema, CRP: C-reactive protein, ESR: Erythrocyte sedimentation rate 


\begin{tabular}{|c|c|c|c|c|c|}
\hline IBP feature & SPARCC SIJ BME & $\begin{array}{l}\text { SPARCC SIJ } \\
\text { Fat Metaplasia }\end{array}$ & $\begin{array}{l}\text { SPARCC SIJ } \\
\text { Erosion }\end{array}$ & $\begin{array}{l}\text { SPARCC SIJ } \\
\text { Backfill }\end{array}$ & $\begin{array}{l}\text { SPARCC SIJ } \\
\text { Ankylosis }\end{array}$ \\
\hline Night pain & $p=0.938, r=-0.009$ & $p=0.178, r=0.149$ & $p=0.361, r=0.101$ & $p=0.936, r=0.009$ & $p=0.413, r=0.090$ \\
\hline Duration above 3 months & $p=0.188, r=-0.098$ & $p=0.355, r=0.046$ & $p=0.264, r=-0.070$ & $p=0.286, r=0.063$ & $p=0.449, r=0.014$ \\
\hline Morning stiffness & $p=0.197, r=-0.142$ & $p=0.270, r=0.122$ & $p=0.059, r=-0.207$ & $p=0.275, r=-0.121$ & $p=0.048^{*}, r=-0.218$ \\
\hline Improvement with exertion & $p=0.914, r=-0.014$ & $p=0.101, r=-0.180$ & $p=0.806, r=-0.027$ & $p=0.214, r=-0.137$ & $p=0.114, r=-0.174$ \\
\hline Began before the age of 40 & $p=0.584, r=-0.061$ & $p=0.564, r=-0.064$ & $p=0.696, r=-0.043$ & $p=0.604, r=-0.057$ & $p=0.235, r=0.131$ \\
\hline
\end{tabular}

that physicians can reduce the disease progression with early treatment by encouraging the evaluation of SIJ MRG in patients with IBP characteristics. However, Arnbak et al. (21) examined the relationship between IBP characteristics and MRI findings in the spine and SIJ and they found a higher association between night pain and SIJ sclerosis and found that structural changes are more consistently related with pain characteristics than BME findings are associated with active inflammation. Similarly, when we compare MRI examinations based on SPARCC scoring and IBP characteristics based on anamnesis in our study, we found that morning stiffness was significantly correlated with the presence of ankylosis. The contradictory study results suggest that there is a weak correlation between the acute or structural findings of the axSpA-related MRI and IBP parameters, but a more consistent correlation between structural findings and IBP can be attributable to the fact that chronic MRI manifestations represent a more severe inflammatory process. Moreover, during the diagnoses made herein, we found that most patients had a high total IBP score (mean score; $4.0 \pm 0.7$ ), whereas there was no difference in the total IBP score of patients with and without acute or structural lesions in SIJ MRI.

The current approach to axSpA treatment is to intervene at disease onset, in which the response to treatment is the greatest before the appearance of radiographic sacroiliitis (22). Therefore, it is important to demonstrate inflammation with MRI before the detection of radiographic changes and identify patients with axSpA with a higher risk of progression to AS. Blachier et al. (23) showed that structural radiological lesions in early axSpA were positively correlated with the CRP level and active inflammation seen in the MRI of SIJ. A recent study by Min et al. (24) found that has a higher CRP level and ESR than non-radiographic axSpA. Similarly, ESR levels were found to be significantly higher in axSpA patients with structural lesions (backfill and ankylosis) in our study. The aforementioned findings support that the acute inflammatory condition is more common in AS patients in terms of biochemical parameters and MRI findings compared to non-radiographic axSpA. Although the severity of sacroiliitis was not evaluated radiographically in our study, the mean basal SIJ MRG inflammation score was 18.4 according to the SPARCC scoring and higher than the previously reported values in non-radiographic axSpA studies (7.4 and 10.0, respectively) $(25,26)$. Again, the mean basal SIJ MRI inflammation score in our study was $>14.0$ which was same as the score in the group of AS patients in the study by Min et al. (24), and this difference might be attributable to the inclusion of more patients with advanced structural lesions (presence of SIJ ankylosis is $82.1 \%$ ). This, in turn, supports studies in which the severity of sacroiliitis on plain radiography was positively correlated with the inflammatory score of SIJ MRI. Moreover, the symptom duration in non-radiographic axSpA and AS in the study by Min et al. (24) was 1 and 2 years, respectively and the symptom duration in our study was 3.3 years; the symptom duration was significantly longer in patients with SIJ erosion and ankylosis than those who did not which may be another reason for the large number of structural lesions in our study.

SIJ radiography is recommended as the first imaging modality to diagnose sacroiliitis as part of axSpA. Since it can be identified even in patients whose structural lesions are radiographically normal on SIJ MRI, it is better to use MRI instead of radiography to identify structural lesions as well as inflammation because MRI has higher sensitivity than radiography. Indeed, BME is evident on MRI several years prior to the development of structural changes visible on radiograph (27). Structural lesions are also generally not observed on radiographs for more than five years from the onset of the disease (28). In addition, as shown in our study, clinical and laboratory characteristics have been determined to be associated with advanced structural lesions. In conclusion, MRI can identify patients who can avoid further radiographic progress with early detection of the acute and early-stage structural lesions in SIJ and diagnostic abilities of SpA can be enhanced by adding MRI in the diagnostic workup. 


\section{Study Limitations}

Our research has certain limitations. First, since the patients in our study were admitted to the Physical Medicine and Rehabilitation outpatient clinic, this group might include patients with advanced MRI lesions. Second, the severity of sacroiliitis was not evaluated radiographically due to the lack of plain radiographical images of the patients. Third, because it was a retrospective examination, it contains only basic data and information indicating the activity of the disease, such as Bath AS Disease Activity Index, is missing. Finally, the high level of intra-reader variability cannot be ignored because the MRI assessment and calculation of SPARCC scores were performed by a single radiologist.

\section{Conclusion}

According to the results of this study, ESR values and symptom duration are statistically higher in axSpA patients with structural lesions in SIJ MRI than those who did not and morning stiffness was significantly correlated with the presence of ankylosis. In our study, clinical and laboratory characteristics were associated with structural lesions rather than acute inflammation in MRI findings. Further research is needed to correlate specific MRI findings with clinical disease characteristics.

\section{Acknowledgements}

The authors thank Deniz Yakar Mutlu for MRI assessments in this study.

\section{Authorship Contributions}

Concept: M.C., Design: M.C., T.A., Data Collection or Processing: M.C., T.A. Analysis or Interpretation: T.A. Literature Search: T.A. Writing: M.C.

Conflict of Interest: This study was conducted as a master's thesis. The authors confirm that this article's content has no conflicts of interest.

Financial Disclosure: The authors declared that this study received no financial support

\section{References}

1. Cakır N, Pamuk ÖN, Derviş E, et al. The prevalences of some rheumatic diseases in western Turkey: Havsa study. Rheumatol Int 2012;32:895-908.

2. Baraliakos X, Tsiami S, Redeker I, et al. Early recognition of patients with axial spondyloarthritis-evaluation of referral strategies in primary care. Rheumatology (Oxford) 2020;59:3845-52.

3. Gökşenoğlu G, Buğdaycı D, Paker N, Yıldıım MA, Etli Ö. The prevalence of comorbidity and predictors in ankylosing spondylitis. Turk J Phys Med Rehabil 2018;65:132-8.
4. van Hoeven L, Luime J, Han H, Vergouwe Y, Weel A. Identifying axial spondyloarthritis in Dutch primary care patients, ages 20-45 years, with chronic low back pain. Arthritis Care Res (Hoboken) 2014;66:446-53.

5. Masson Behar V, Dougados M, Etcheto A, et al. Diagnostic delay in axial spondyloarthritis: A cross-sectional study of 432 patients. Joint Bone Spine 2017;84:467-71.

6. Sieper J, Rudwaleit M, Baraliakos X, et al. The Assessment of SpondyloArthritis international Society (ASAS) handbook: a guide to assess spondyloarthritis. Ann Rheum Dis 2009;68:144.

7. Protopopov M, Sieper J, Haibel H, Listing J, Rudwaleit $M$, Poddubnyy $D$. Relevance of structural damage in the sacroiliac joints for the functional status and spinal mobility in patients with axial spondyloarthritis: results from the German Spondyloarthritis Inception Cohort. Arthritis Res Ther 2017;19:240.

8. laremenko O, Shynkaruk I, Fedkov D, laremenko K, Petelytska L. Bone turnover biomarkers, disease activity, and MRI changes of sacroiliac joints in patients with spondyloarthritis. Rheumatol Int 2020;40:2057-63.

9. Schwartzman M, Maksymowych WP. Is There a Role for MRI to Establish Treatment Indications and Effectively Monitor Response in Patients with Axial Spondyloarthritis? Rheum Dis Clin North Am 2019;45:341-58.

10. Rudwaleit $M$, Jurik $A G$, Hermann $K G$, et al. Defining active sacroilitis on magnetic resonance imaging (MRI) for classification of axial spondyloarthritis: a consensual approach by the ASAS/OMERACT MRI group. Ann Rheum Dis 2009;68:1520-7.

11. Mandl P, Navarro-Compán V , Terslev L, et al. EULAR recommendations for the use of imaging in the diagnosis and management of spondyloarthritis in clinical practice. Ann Rheum Dis 2015;74:1327-39.

12. Rudwaleit M, Metter A, Listing J, Sieper J, Braun J. Inflammatory back pain in ankylosing spondylitis: a reassessment of the clinical history for application as classification and diagnostic criteria. Arthritis Rheum 2006;54:569-78.

13. Weisman MH, Witter JP, Reveille JD. The prevalence of inflammatory back pain: population-based estimates from the US National Health and Nutrition Examination Survey, 2009-10. Ann Rheum Dis 2013;72:369-73.

14. Reveille JD. Epidemiology of spondyloarthritis in North America. Am J Med Sci 2011;341:284-6.

15. Landewé RB, Hermann KG, van der Heijde DM, et al. Scoring sacroiliac joints by magnetic resonance imaging. A multiplereader reliability experiment. J Rheumatol 2005;32:2050-5.

16. Maksymowych WP, Inman RD, Salonen $D_{\text {, et al. }}$ Spondyloarthritis research Consortium of Canada magnetic resonance imaging index for assessment of sacroiliac joint inflammation in ankylosing spondylitis. Arthritis Care Res 2005;53:703-9. 
17. Kivity S, Gofrit SG, Baker FA, et al. Association between inflammatory back pain features, acute and structural sacroiliitis on MRI, and the diagnosis of spondyloarthritis. Clin Rheumatol 2019;38:1579-85.

18. van Onna $M$, Jurik $A G$, van der Heijde $D$, et al. HLA-B27 and gender independently determine the likelihood of a positive MRI of the sacroiliac joints in patients with early inflammatory back pain: a 2-year MRI follow-up study. Ann Rheum Dis 2011;70:1981-5.

19. Van den Berg $R$, de Hooge $M$, Rudwaleit $M$, et al. ASAS modification of the Berlin algorithm for diagnosing axial spondyloarthritis: results from the SPondyloArthritis Caught Early (SPACE)-cohort and from the Assessment of SpondyloArthritis international Society (ASAS)-cohort. Ann Rheum Dis 2013;72:1646-53.

20. Braun J, Inman R. Clinical significance of inflammatory back pain for diagnosis and screening of patients with axial spondyloarthritis. Ann Rheum Dis 2010;69:1264-8.

21. Arnbak B, Jurik AG, Jensen TS, Manniche C. Association Between Inflammatory Back Pain Characteristics and Magnetic Resonance Imaging Findings in the Spine and Sacroiliac Joints. Arthritis Care Res (Hoboken) 2018;70:24451.

22. Ward MM, Deodhar A, Akl EA, Lui A, et al. American College of Rheumatology/Spondylitis Association of America/Spondyloarthritis Research and Treatment Network 2015 Recommendations for the Treatment of Ankylosing
Spondylitis and Nonradiographic Axial Spondyloarthritis. Arthritis Rheumatol 2016;68:282-98.

23. Blachier $M$, Canouï-Poitrine $F$, Dougados $M$, et al. Factors associated with radiographic lesions in early axial spondyloarthritis. Results from the DESIR cohort. Rheumatology (Oxford) 2013;52:1686-93.

24. Min HK, Cho H, Park SH. Baseline severity of sacroiliitis can predict acute inflammatory status of sacroiliac joint in early axial spondyloarthritis of male patients: a cross sectional study. BMC Musculoskelet Disord 2019;20:144.

25. Rusman T, John MB, van der Weijden MAC, et al. Presence of active MRI lesions in patients suspected of non-radiographic axial spondyloarthritis with high disease activity and chance at conversion after a 6-month follow-up period. Clin Rheumatol 2020;39:1521-9.

26. Maksymowych WP, Dougados $M$, van der Heijde $D$, et al. Clinical and MRI responses to etanercept in early nonradiographic axial spondyloarthritis: 48-week results from the EMBARK study. Ann Rheum Dis 2016;75:1328-35.

27. Dincer U, Cakar E, Kiralp MZ, Dursun H. Diagnosis delay in patients with ankylosing spondylitis: possible reasons and proposals for new diagnostic criteria. Clin Rheumatol 2008;27:457-62.

28. Guglielmi G, Cascavilla A, Scalzo G, Carotti M, Salaffi F, Grassi W. Imaging findings of sacroiliac joints in spondyloarthropathies and other rheumatic conditions. Radiol Med 2011;116:292301. 